\title{
Avaliação da visão funcional em crianças com deficiência visual e múltipla deficiência como estratégia de apoio para professores e responsáveis
}

\author{
Isabela Maria Reis Barbosa* \\ Heloisa Gagheggi Ravanini Gardon Gagliardo** \\ Marilda Moraes Garcia Bruno*** \\ Maria Elisabete Rodrigues Freire Gasparetto****
}

\section{Resumo}

A avaliação da visão funcional é um importante recurso para oferecer informações sobre a condição visual das crianças com múltipla deficiência, tendo em vista que grande parte desse público não recebe diagnóstico visual em tempo oportuno devido às dificuldades enfrentadas para a realização da avaliação oftalmológica. A presente pesquisa teve como objetivo avaliar a visão funcional de crianças com deficiência visual associada à múltipla deficiência, por meio do método de observação do comportamento e do teste Lea Gratings. Foi realizado um estudo de caso múltiplo com abordagem qualitativa, com cinco crianças que apresentavam deficiência visual associada à múltipla deficiência. Para a coleta de dados, foi realizada entrevista com os responsáveis e com os professores e avaliada a visão funcional das crianças participantes. $\mathrm{Na}$ avaliação da visão funcional constatou-se que todas as crianças apresentavam valores de acuidade de grades binocular bem abaixo dos valores padrôes estabelecidos pela literatura, entretanto, possuíam visão funcional útil para o desempenho de tarefas cotidianas e escolares. Foi elaborado um instrumento denominado "suporte informacional", que foi entregue aos participantes após o término da pesquisa, com dados sobre o resultado da avaliaçáo da visão funcional das crianças, bem como informações sobre a visão, deficiência visual associada à múltipla deficiência e avaliaçâo da visão funcional. $\mathrm{O}$ estudo demonstrou que a avaliação da visão funcional foi relevante para subsidiar a proposição de orientaçóes adequadas para o desenvolvimento das açôes dos responsáveis e das práticas pedagógicas dos professores com os alunos que participaram da pesquisa.

Palavras-chave: Avaliação; Visão; Inclusão educacional.

\footnotetext{
* Mestra em Saúde, Interdisciplinaridade e Reabilitação, pela Faculdade de Ciências Médicas da Universidade Estadual de Campinas, Campinas, São Paulo, Brasil.

** Professora Doutora do Departamento de Desenvolvimento Humano e Reabilitação e do Programa de Pós Graduação Saúde, Interdisciplinaridade e Reabilitação da Faculdade de Ciências Médicas da Universidade Estadual de Campinas, Campinas, São Paulo, Brasil.

*** Docente do Programa de Pós Graduação em Educação da Faculdade de Educação pela Universidade Federal de Grande Dourados, Dourados, Mato Grosso do Sul, Brasil.

**** Professora Doutora do Departamento de Desenvolvimento Humano e Reabilitação e do Programa de Pós Graduação Saúde, Interdisciplinaridade e Reabilitação da Faculdade de Ciências Médicas da Universidade Estadual de Campinas, Campinas, São Paulo, Brasil.
} 


\section{Assessment of functional vision in children with visual disability and multiple disabilities as support strategy for teachers and responsible}

\section{Abstract}

The assessment of functional vision is an important resource to provide information about the visual condition of children with multiple disabilities, considering that much of this public do not receive visual diagnosis in timely period, due to difficulties to carry out the ophthalmologic evaluation. This study aimed to assess the functional vision of children with visual impairment associated to multiple disabilities, through the observation method of behavior and Lea Gratings test. It was realized a multiple case study with a qualitative approach, with 05 children who had presented visual impairment associated to multiple disabilities. To data collect, it was made interview with the parents and teachers about visual and comportamental caracteristics and about the scholling process of participating children, then of assessment functional vision of the children. The results of the assessment of functional vision showed that the participants children presented low values of visual acuity of binocular grids in comparision to literature, however, showed that all children had functional vision to perform daily tasks and school. It was elaborated an instrument called "informational support", which was delivered to participants after completion of the study, containing informations about vision, visual disability in association with multiple disabilities and assessment of functional vision. The study showed that the evaluation of functional vision was relevant to subsidize proposals for appropriate guidance for the development of the actions of the parents and for the development of pedagogical practices of teachers with children who participated in the survey.

Keywords: Assessment; Vision; Educational inclusion.

\section{Introdução}

A múltipla deficiência, apesar de ser, frequentemente, caracterizada como o conjunto de duas ou mais deficiências associadas de ordem física, sensorial e mental, emocional e comportamento social, se constitui pelo desenvolvimento, pelas possibilidades funcionais, de comunicação, de interação social e de aprendizagem das pessoas acometidas (CARVALHO, 2000).

A etiologia da múltipla deficiência é variada e está associada a fatores pré-natais, perinatais e pós-natais (ARAÓZ, 2010). Nobre et al. (2009) referem que o nível de gravidade dos comprometimentos em crianças multideficientes têm aumentado nos últimos anos, em decorrência, principalmente, do avanço da medicina em relação à prematuridade.

De acordo com Remigio et. al (2006), as crianças com múltipla deficiência apresentam maior proporção (60 a 90\%) de alterações oculares em relação às crianças sem deficiência. 
Devido à gravidade neurológica dos casos, a dificuldade de acesso aos centros especializados, a dificuldade para definição de diagnósticos, entre outros motivos, a maioria da população com múltipla deficiência é encaminhada para uma avaliação oftalmológica e para o serviço de estimulação visual tardiamente, o que, consequentemente, diminui as possibilidades de desenvolvimento visual em período adequado, além de desencadear alteraçôes em todos os aspectos do desenvolvimento (NOBRE et al, 2009; GATO et al, 2008).

Este estudo, que teve como objetivo a avaliação da visão funcional em crianças com deficiência visual associada à múltipla deficiência é fruto da Dissertação de Mestrado "Avaliação da visão funcional em crianças com deficiência visual e múltipla deficiência: subsídios para orientaçôes aos responsáveis e aos professores". A pesquisa apresentou metodologia na perspectiva do desenvolvimento humano ecológico e foi desenvolvida por meio do método de observaçáo do comportamento em situaçóes cotidianas e do teste Lea Gratings. Em resultados obtidos, foi apresentada a ação desenvolvida para a orientação aos familiares e professores das crianças com múltipla deficiência.

\section{Avaliação visual da criança com deficiência visual e múltipla deficiência}

Zimermmann (2013) afirma que a avaliação visual da criança requer técnicas e instrumentos específicos, condizente com a faixa etária da mesma. No caso de analfabetos, crianças pré-verbais ou não verbais, faz-se necessário o uso de avaliaçóes específicas.

Salomão (2007), citando Dobson \& Teller (1978) e Day (1997), refere que a partir da década de 1970, foram introduzidos dois métodos - olhar preferencial (OP) e potenciais visuais evocados (PVE) - para definir o desenvolvimento da visão em bebês e crianças pré-verbais, possibilitando a compreensão do desenvolvimento normal e anormal da visão.

As avaliaçóes pautadas nas Técnicas do Olhar Preferencial mensuram a acuidade visual de grades. Esse tipo de acuidade visual se desenvolve nos primeiros anos de vida (SALOMÃO, 2007) e alcança aproximadamente $25 \mathrm{cpg}$ (valor muito próximo a do adulto, que corresponde a $30 \mathrm{cpg}$ ) aos 04 anos (MAYER et al, 1995) e se baseia na habilidade do sujeito em resolver padróes listrados ou em formato de tabuleiro de xadrez visual.

Os testes de resolução mais bem sucedidos e frequentemente utilizados são os que empregam o procedimento do olhar preferencial, destacando-se o teste de cartóes de acuidade visual de Teller e o teste das raquetes de Lea Hyvarinen (CÔRREA, 2011). Baseando-se na referência bibliográfica, o teste das raquetes de Lea Hyvarinen, Lea Gratings, foi utilizado neste estudo, por tratar-se de instrumento simples e coerente e fornecer medidas comportamentais e consistentes do desempenho visual das crianças (YUDCOVITCH et al., 2004). O teste Lea Gratings constituiu-se também como um instrumento confiável, de fácil acesso, baixo custo e passível de aplicabilidade por profissionais que atuam na área da educação e na área da saúde. 
Tabuse \& Cronemberger (2010) afirmaram que, além desses testes, a avaliação da observação do comportamento se estabelece como um importante instrumento de identificação das limitaçôes e potencialidades visuais de crianças em níveis iniciais de desenvolvimento, sendo utilizada para observar a fixação e o seguimento visual referentes a foco de luz e/ou objetos coloridos. Marques \& Mendes (2014) referem que os aspectos a serem observados, na avaliação da visão funcional são a fixação visual, os movimentos oculares e o uso da visão para as tarefas e atividades cotidianas.

Topor (2009) refere que quando não há uma avaliação oftalmológica, a avaliação da visão funcional pode ser o primeiro passo para apontar a necessidade de um exame clínico. De acordo com Bruno (2011), a avaliaçẫo da visão funcional é um procedimento de avaliação qualitativa e contínua, que objetiva estimar o uso da visão, realizada por meio da observação informal e natural da criança em todas as situaçôes de vida e nas atividades funcionais. Por meio da avaliação da visão funcional, é possível conhecer as condiçóes que favorecem a habilidade funcional de utilização da visão, bem como os recursos sensoriais remanescentes (FAVILLA et al, 2014).

Muitos pesquisadores têm utilizado testes informais que avaliam características do funcionamento visual na perspectiva da avaliação da visão funcional (ISAAC et al, 2003). Tais recursos avaliativos não possuem formalizaçáo quanto aos materiais utilizados, mas a maioria das pesquisas apontam que os materiais mais comuns para composição de recursos avaliativos são brinquedos e objetos de uso cotidiano dos sujeitos, com cores de alto-contraste e luminosidade ((HOUSTON-PRICE \& NAKAI, 2004; BRUNO, 2005; ILLET, 2010; ZIMMERMANN, 2013).

Os instrumentos utilizados para a verificação da habilidade visual, fornecem dados sobre o funcionamento visual da visão de perto e de longe. Por meio dos materiais utilizados na avaliação da observação do comportamento, é possível observar funçóes visuomotoras e a esfera visual. $\mathrm{O}$ estimulo visual desencadeado a partir de luz direta sobre objetos sonoros, pode propiciar a demonstraçáo de preferências por algum tipo de som que pode ser notada por meio de uma reação social. A associação de estímulos visuais à exploração tátil durante a manipulação de objetos favorece a integração das diferentes informaçóes sensoriais e motiva a criança à exploração (FAVILLA et al, 2014).

Isaac et. al (2003) afirmam que indivíduos com prejuízos visuais causados por lesão cerebral ou cortical apresentam características visuais diferentes de indivíduos com prejuízos visuais oculares. Em complemento, Bruno (2005) refere que essa diferenciação ocorre principalmente nas habilidades para processar informaçôes visuais e na formação de conceitos. Marques \& Mendes (2014) afirmam que a avaliação clínica na deficiência visual cortical é um grande desafio, "devido à variabilidade e complexidade das possíveis alteraçóes visuais aliadas aos diferentes e múltiplos comprometimentos associados que prejudicam as possibilidades de resposta-açấo".

As crianças com múltipla deficiência podem apresentar vários comprometimentos que nem sempre estão evidentes, por isso, se faz necessária a investigação da condição visual para orientar os responsáveis e professores, visando subsidiar o desenvolvimento de um programa educacional. 
Bruno (2008) afirma que são acentuados os obstáculos referentes a educação de crianças com deficiência visual e múltipla, pelo predomínio da abordagem clínica e terapêutica, com modelos mecanicistas de reeducação e treinamento visual, cuja ênfase recai na modificação do sujeito para que se adapte ao meio. Os fatores que desencadeiam essas situaçóes decorrem da falta de estudos sobre a avaliaçáo do processo de desenvolvimento, aprendizagem e das necessidades específicas e educacionais dessa população.

Silva-Porta et. al (2016) referem que, as adaptações curriculares devem se basear nos Parâmetros Curriculares Nacionais e atuar diante das dificuldades dos educandos público-alvo da educação especial, englobando os alunos com múltipla deficiência.

O Conselho Internacional de Educação de Pessoas com Deficiência Visual (ICEVI, 1992) indica que a proposta pedagógica mais adequada para a criança com múltipla deficiência baseia-se no currículo funcional - fundamentado no conceito de Ecologia do Desenvolvimento Humano -, em que o planejamento prevê o ensino das habilidades necessárias para a possibilidade da vida autônoma.

O enfoque ecológico pauta-se na aprendizagem significativa e útil e considera importante que o currículo seja desenvolvido no ambiente natural de convívio do educando. O currículo deve estar voltado à realidade do aluno, partindo do levantamento de informaçóes sobre essa realidade e sobre suas formas próprias de agir no mundo. Ressalta-se que, o processo educacional da criança com múltipla deficiência depende também do envolvimento da comunidade, possibilitando a participaçáa e interação da criança nos diversos espaços e nas diversas práticas sociais (SIAULYS et. al, 2011). Assim como trazido por Bruno (2006, p. 46), "tanto o processo de avaliação como o currículo chamam-se "funcionais" porque buscam meios úteis e funcionais para melhorar o desempenho global, o desenvolvimento de competência social, o acesso ao conhecimento, à cultura e as formas de trabalho valorizadas pela comunidade a qual os educandos pertencem”.

\section{Objetivos}

Avaliar a visão funcional de crianças que apresentavam múltipla deficiência, visando verificar presença de resíduo visual para fornecer subsídios e orientaçóes aos responsáveis por essas crianças e aos professores.

Investigar se os responsáveis e os professores receberam informaçóes e orientaçôes sobre a condição visual das crianças.

\section{Materiais e métodos}

\section{Desenho do estudo}

A metodologia do estudo configurou-se como estudo de casos múltiplos de crianças com deficiência visual associada à múltipla deficiência, inseridas no ensino regular, que envolveu a participação de responsáveis e educadores das mesmas, com o propósito de conhecer e refletir sobre a importância da avaliação da visão funcional para o planejamento de estratégias de intervenção e apoio educacional. 
Isabela Maria Reis Barbosa - Heloísa Gagheggi Ravanini Gardon Gagliardo - Marilda

Moraes Garcia Bruno - Maria Elisabete Rodrigues Freire Gasparetto

\section{Aspectos éticos}

A pesquisa foi aprovada pelo Comitê de Ética em Pesquisa da Universidade Estadual de Campinas, pelo CAEE: 22763713.2.0000.5404.

Os responsáveis pelos participantes e os professores dos alunos foram convidados a participar da pesquisa. Os objetivos foram informados pela pesquisadora e foi solicitado o consentimento por escrito, por meio do Termo de Consentimento Livre e Esclarecido

Foi solicitado à Secretaria de Educação da Prefeitura Municipal da Estância Balneária de Caraguatatuba, autorização para que a pesquisadora pudesse realizar a pesquisa nos locais acima citados.

\section{Amostra}

Participaram do estudo cinco crianças com deficiência visual associada à múltipla deficiência, com faixa etária entre três e dez anos (média das idades de 6,6 anos), de ambos os sexos (sendo quatro meninos e uma menina), recrutados em unidades escolares regulares do município de Caraguatatuba-SP. As crianças foram selecionadas e indicadas por intermédio da equipe de profissionais do setor de Educação Inclusiva do município de Caraguatatuba.

Para fins de inclusão no estudo, foram consideradas as crianças que segundo a pesquisa documental e relato dos responsáveis apresentavam baixa visão devido à atrofia do nervo óptico, estrabismo sensorial (secundário à baixa visão) e miopia (dificuldade para ver de longe) e duas crianças que ainda estavam sendo diagnosticadas, mas, não forneciam resposta visual quando eram apresentados objetos sem adaptação e sem modificação ambiental.

Os critérios de caracterização da múltipla deficiência, no presente estudo, consideraram a existência de limitaçôes motoras graves e severas e a não existência de comunicação por meio da expressão verbal. A classificação motora da disfunção da mobilidade funcional dos casos foram realizadas pela pesquisadora, utilizando o "Sistema de Avaliação da Função Motora Grossa" (GMFCS), que é um instrumento de classificação por níveis de mobilidade funcional em crianças com Paralisia Cerebral. Apesar de ser um instrumento desenvolvido para a classificação de indivíduos que apresentam Paralisia Cerebral, entendeu-se possível utilizá-lo para avaliar as condiçóes motoras dos participantes do estudo, considerando que muitos deles não possuem diagnóstico concluído e considerando ainda, que os quadros clínico e terapêutico se assemelham ao da Paralisia Cerebral, em todos os casos.

Participaram do estudo, ainda, cinco responsáveis pelas crianças e os cinco professores responsáveis pelas turmas regulares frequentadas pelas crianças. Os professores tinham formação na área de pedagogia, mas não detinham conhecimentos específicos em relação à atuação na múltipla deficiência. 


\section{Instrumentos de avaliação e coleta de dados}

Primeiramente, foi realizada anamnese e entrevista com os responsáveis das crianças (quatro mães e uma avó), a fim de coletar as características pessoais, o histórico da deficiência das crianças, o recebimento de informaçôes e orientações médicas e de outros profissionais sobre a condição visual e a participação da criança no Atendimento Educacional Especializado (AEE). A primeira etapa da entrevista foi baseada na avaliação desenvolvida por Bruno (2005).

Posteriormente, foi solicitado aos professores das crianças participantes da pesquisa que informassem o recebimento de informaçóes médicas e informaçóes de outros profissionais sobre a condição visual das crianças.

Para avaliar a visão funcional das crianças optou-se por um modelo quantitativo que verificou a acuidade visual de grades, por meio do teste Lea Gratings, elaborado por Lea Hyvarinen (figura 1). Utilizando-se de um modelo qualitativo, realizou-se também a avaliação da visão funcional por meio da observação do comportamento, utilizando-se um roteiro de avaliação, elaborado pela própria pesquisadora, mas, com embasamento no roteiro da avaliação da visão funcional, desenvolvida por Gagliardo, em 2005, e que é utilizada no setor de Terapia Ocupacional do Programa Habilitação e Reabilitação de Crianças com Deficiência Visual do Centro de Estudos e Pesquisas em Reabilitação "Prof. Dr. Gabriel Porto" da Universidade Estadual de Campinas.

Durante todo o processo de avaliação da visão, as crianças permaneceram sentadas em cadeira de rodas ou em colchonete, com auxílio de terceiros. As avaliaçóes foram realizadas em sessão única, tendo duração média aproximada de 40 minutos.

Visando conhecer o valor da acuidade visual das crianças, foi aplicado o método de acuidade visual de grades, teste Lea Gratings ${ }^{2}$, seguindo rigorosamente as instruçóes que foram indicadas por Pimenta (2012). Inicialmente as raquetes foram apresentadas à distância de $28 \mathrm{~cm}$; quando as respostas dos participantes mostravamse positivas as raquetes eram distanciadas e quando as respostas dos participantes mostravam-se negativas, as raquetes eram aproximadas. 
Figura 1 - Raquetes do teste Lea Gratings (PIMENTA, 2013)

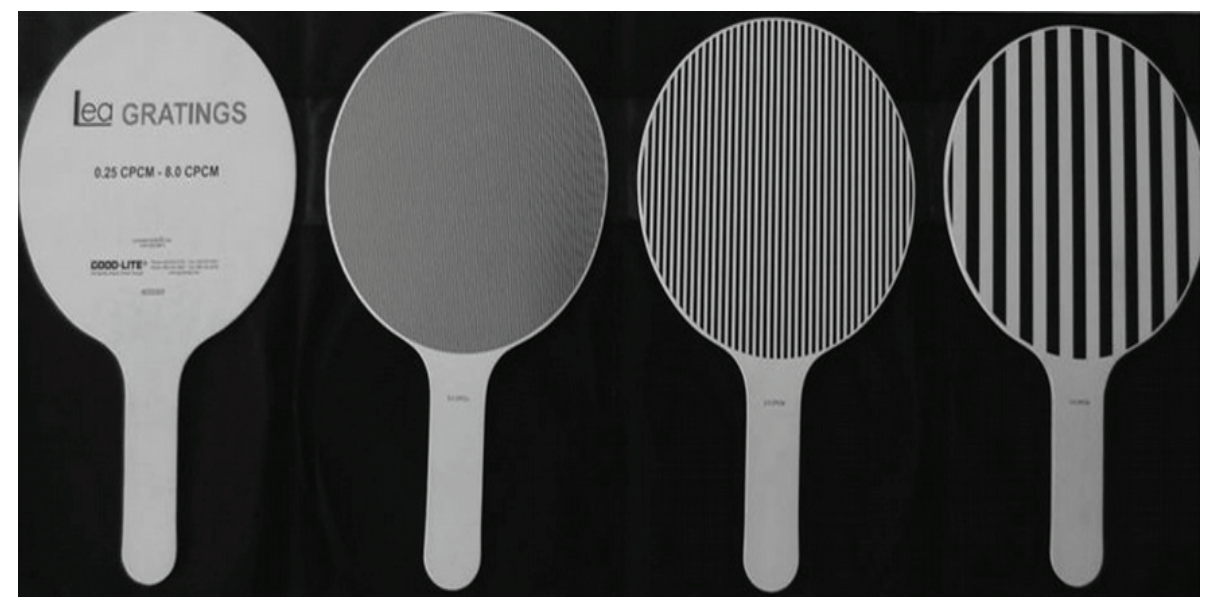

A avaliaçáo para verificar presença de resíduo visual também foi realizada por meio da observaçáo do comportamento e objetivou apreender a resposta visual das crianças com deficiência visual associada à múltipla deficiência. Para sua aplicação, a pesquisadora fez uso de lanterna de pilhas comuns com foco pequeno $(3 \mathrm{~cm}$ de diâmetro), brinquedos diversos (coelho de pelúcia de diâmetro aproximado de $14 \mathrm{~cm}$, carrinho de diâmetro aproximado de $8 \mathrm{~cm}$ e boneca de diâmetro aproximado de 3,5 $\mathrm{cm}$ ) e suporte branco para apresentação dos objetos, a fim de criar contraste adequado para facilitar a visualização pelos participantes, conforme apresentado na figura 2 .

Figura 2 - Objetos utilizados na avaliaçáo, por meio da observação do comportamento visual

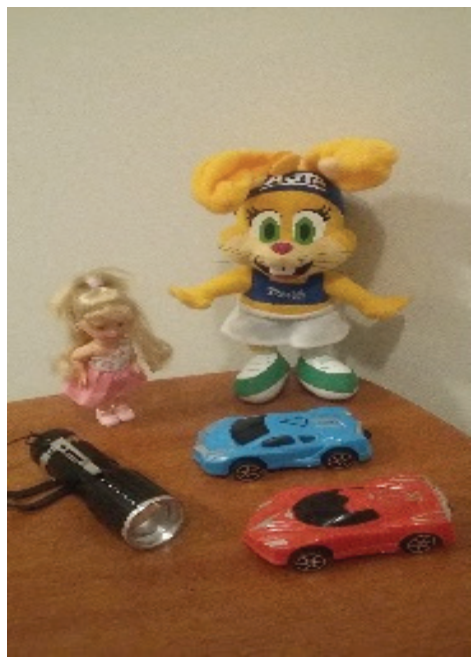




\section{Resultados e discussão}

\section{Caracterização dos participantes}

Quadro 1 - Caracterizaçâo dos participantes

\begin{tabular}{|c|c|c|c|c|c|c|}
\hline & Idade(anos) & Sexo & $\begin{array}{l}\text { Escolari- } \\
\text { dade }\end{array}$ & $\begin{array}{l}\text { Hipótese } \\
\text { Diagnóstica }\end{array}$ & $\begin{array}{l}\text { Diagnótico } \\
\text { Visual }\end{array}$ & $\begin{array}{l}\text { Classificaçáa } \\
\text { GMFCS }\end{array}$ \\
\hline Criança 1 & 10 & Masculino & $3^{\circ}$ ano EFI & $\begin{array}{l}\text { Encefalopatia } \\
\text { Crônica Náo } \\
\text { Progresiva da } \\
\text { infanância }\end{array}$ & Inconclusivo & Nível V \\
\hline Criança 2 & 9 & Masculino & $4^{\circ}$ ano EFI & $\begin{array}{l}\text { Atraso no de- } \\
\text { senvolvimen- } \\
\text { to neuropsi- } \\
\text { comitor }\end{array}$ & $\begin{array}{l}\text { Estrabismo sen- } \\
\text { sorial e miopia }\end{array}$ & Nível V \\
\hline Criança 3 & 7 & Masculino & $1^{\circ}$ ano EFI & $\begin{array}{l}\text { Sindrome de } \\
\text { Dandy Walker }\end{array}$ & $\begin{array}{l}\text { Atrofia do } \\
\text { nervo óptico }\end{array}$ & Nível IV \\
\hline Criança 4 & 3 & Feminino & $\begin{array}{l}\text { Maternal } \\
\text { II EI }\end{array}$ & Hidrocefalia & Inconclusivo & Nível V \\
\hline Criança 5 & 4 & Masculino & $\begin{array}{l}\text { Maternal } \\
\text { II EI }\end{array}$ & $\begin{array}{l}\text { Encefalopatia } \\
\text { Crônica Não } \\
\text { Progresiva da } \\
\text { infanância }\end{array}$ & $\begin{array}{l}\text { Atrofia do } \\
\text { nervo óptico }\end{array}$ & Nível V \\
\hline
\end{tabular}

Verificou-se que apenas três participantes possuíam diagnóstico visual conclusivo; entretanto, um dos participantes apresentava, apenas, diagnóstico de estrabismo e erro refracional, apesar de apresentar evidências de deficiência visual cortical. Esses dados vão ao encontro dos achados da literatura, que comprovam que o diagnóstico das alteraçôes visuais ocorre tardiamente, em grande parte dos casos de crianças com deficiência visual associada à múltipla deficiência (NOBRE et al, 2009; GATO et al, 2008), em consequência da alteração visual ser considerada de menor relevância frente a outros diagnósticos ou, ainda, pela dificuldade de acesso a profissionais especializados (REMIGIO et. al, 2006).

A classificação do "Sistema de Avaliação da Função Motora Grossa" mostrou que quatro dos cinco participantes apresentavam limitaçóes motoras severas. Os achados podem estar relacionados ao fato de que as informaçóes captadas pelos receptores do equipamento sensorial fornecem estímulos aos movimentos, consciência do esquema corporal e, consequentemente, base às futuras aquisiçóes motoras (FIGUEIRA, 2000). Ademais, a condução da avaliação da visão funcional, no caso dos participantes que apresentaram limitaçóes motoras severas, foi mais difícil do que a condução da avaliação na criança que foi classificada com menor grau de comprometimento da função motora grossa. Esses dados podem ser relacionados às afirmaçóes de Dutton (2002) e Veitzman (2000), de que quanto mais afetado o cérebro, mais difícil é a avaliação. 
Apesar de todas as crianças terem sido avaliadas por um profissional médico oftalmologista, nem todas possuíam diagnóstico visual, o que comprova a afirmação de Remigio et al (2006), de que grande parte dos profissionais médicos têm dificuldades em lidar com crianças com múltipla deficiência. Além disso, nenhuma das crianças havia realizado ou realizava reabilitação visual, o que corrobora com as afirmaçóes de que, apenas, uma parcela ínfima de pessoas com deficiência visual possuem acesso a programas de reabilitação visual.

Apesar de todas as cinco crianças terem realizado avaliação oftalmológica, duas ainda estavam sob investigação oftalmológica e não possuíam o diagnóstico da baixa visual. Tal fato, comprova a afirmaçáo de Remigio et al (2006), de que grande parte dos profissionais médicos têm dificuldades em lidar com crianças com múltipla deficiência. Além disso, nenhuma das crianças havia realizado ou realizava reabilitação visual, o que corrobora com as afirmaçóes de que, apenas, uma parcela ínfima de pessoas com deficiência visual possuem acesso a programas de reabilitação visual. (VEITZMAN, 2000; NAKANAMI \& VASCONCELOS, 2011).

\section{Atendimento Educacional Especializado}

Apenas três das cinco crianças frequentavam o AEE, pois essa modalidade de ensino era disponibilizada, a priori, para as crianças do Ensino Fundamental e as duas crianças que não usufruíam desse serviço frequentavam a Educação Infantil. Fávero et. al (2007) referem que o Atendimento Educacional Especializado deveria estar disponível em todos os níveis de ensino escolar (básico e fundamental), sendo apontado pela Lei de Diretrizes e Bases da Educação Nacional que a Educação Infantil constitui a primeira etapa da Educação Básica (BRASIL, 1996). Roveda (2006) refere ainda que, apesar das recomendaçóes a respeito do trabalho de "estimulação precoce", a formação oferecida aos professores para o trabalho em sala de recursos náo contempla a faixa etária de zero a três anos.

\section{Avaliação da visão funcional}

O quadro 2 apresenta os resultados da avaliação da visão funcional das crianças participantes, por meio do método de observação do comportamento.

Quadro 2 - Avaliação da visão funcional por meio da observação do comportamento

\begin{tabular}{|c|l|l|l|l|l|}
\hline & Criança 1 & Criança 2 & Criança 3 & Criança 4 & Criança 5 \\
\hline Reaçáo à luz & & & & & \\
\hline Reflexo Fotomotor & Apresenta & Apresenta & Apresenta & Apresenta & Apresenta \\
\hline Percepçáo de luz & Apresenta & Apresenta & Apresenta & Apresenta & Apresenta \\
\hline Projeção de luz & Apresenta & Apresenta & Apresenta & Apresenta & Apresenta \\
\hline Fixaçáo Visual & & & & & \\
\hline Firme/Instável & Instável & Instável & Firme & Instável & Instável \\
\hline Diretal Indireta & Indireta & Indireta & Direta & Indireta & Indireta \\
\hline
\end{tabular}




\begin{tabular}{|c|c|c|c|c|c|}
\hline \multicolumn{6}{|l|}{ Seguimento visual } \\
\hline $\begin{array}{l}\text { Movimentos } \\
\text { coordenados }\end{array}$ & Não & Não & Sim & Não & Não \\
\hline Horizontal & Sim & Sim & Sim & Sim & Sim \\
\hline Vertical & Não & Sim & Sim & Sim & Não \\
\hline \multicolumn{6}{|l|}{ Atenção Visual } \\
\hline Apresenta/Mantém & Apresenta & Apresenta & $\begin{array}{l}\text { Apresenta/ } \\
\text { Mantém }\end{array}$ & Apresenta & Apresenta \\
\hline \multicolumn{6}{|l|}{ Campo visual } \\
\hline $\begin{array}{l}\text { Temporal Diretol } \\
\text { Esquerdo/Central }\end{array}$ & $\begin{array}{l}\text { L a t e r a l } \\
\text { direito }\end{array}$ & $\begin{array}{l}\mathrm{L} \text { a t e r a } 1 \\
\text { direito }\end{array}$ & Central & $\begin{array}{l}\text { L a t e r a l } \\
\text { direito }\end{array}$ & $\begin{array}{l}\text { L a t e r a l } \\
\text { direito }\end{array}$ \\
\hline $\begin{array}{l}\text { Superior/Inferiorl } \\
\text { Linha média }\end{array}$ & Inferior & Superior & Inferior & Inferior & Inferior \\
\hline \multicolumn{6}{|l|}{ Acuidade visual } \\
\hline Esfera visual & $30 \mathrm{~cm}$ & $50 \mathrm{~cm}$ & $50 \mathrm{~cm}$ & $30 \mathrm{~cm}$ & $30 \mathrm{~cm}$ \\
\hline $\begin{array}{l}\text { Tamanho do objeto } \\
\text { (dimensão) }\end{array}$ & $14 \mathrm{~cm}$ & $8 \mathrm{~cm}$ & $8 \mathrm{~cm}$ & $14 \mathrm{~cm}$ & $14 \mathrm{~cm}$ \\
\hline
\end{tabular}

Os resultados da avaliação da visão funcional, por meio da observação do comportamento, apontaram que todas as crianças apresentaram reaçôes à luz, tendo sido oferecidas respostas mais rápidas quando os objetos recebiam incidência de luz da lanterna. Além disso, as crianças, em sua maioria, demonstraram maior interesse pelos objetos lúdicos do que pelas raquetes do teste Lea Gratings. Os objetos lúdicos, bem como os objetos de uso cotidiano dos sujeitos, com cores de alto-contraste e luminosidade, têm sido apontados, por inúmeras pesquisas, como os materiais mais comuns para composição de recursos avaliativos, uma vez que possibilitam respostas mais efetivas na avaliação, já que as crianças demonstram maior interesse por esses tipos de materiais ((HOUSTON-PRICE \& NAKAI, 2004; BRUNO, 2005; ILLET, 2010; ZIMMERMANN, 2013).

Apenas a criança 3 apresentou fixação visual firme e direta. $\mathrm{O}$ seguimento visual horizontal foi apresentado por todas as crianças, diferentemente do seguimento visual vertical, apresentado apenas pelas crianças 2,3 e 4 e tendo se mostrado estável apenas pela criança 3, que também foi a única a apresentar movimentos coordenados dos olhos. As funçôes oculomotoras adequadas são um pré-requisito para a criança olhar em direção a um objeto de interesse (HYVARINEN, 1995; NOBRE et. al, 2009). Isso pode justificar o fato da criança 3 ter apresentado melhor desempenho na avaliação do que as demais crianças.

Somente a criança 3 manteve atenção visual, apesar de todas as crianças terem apresentado atenção visual de curto período. Entretanto, foi necessária a utilização de oralização, toques corporais, sorrisos e expressôes faciais durante a realização da 
avaliação, para resgatar a atenção das crianças. De acordo com Hyvarinen (1995), o déficit de atenção visual também se constitui como um fator relacionado, principalmente, aos distúrbios ou danos cerebrais, estando frequentemente associados a uma metade do campo visual (hemi-inatenção).

A maioria das crianças apresentou respostas em mais de um campo visual, mas houve predomínio de um campo visual, durante a avaliação, em todos os casos. Hyvarinen (1995) refere que os escotomas (pontos cegos ou perda de campo irregular), dentro do campo visual, devem ser mensurados com testes clínicos específicos. No entanto, perdas de função nas áreas centrais do campo visual podem tornar-se evidentes quando pequenos objetos ou imagens náo forem, consistentemente, detectados em uma área específica; para isso, faz-se necessária a observação da criança durante a execução de tarefas que requeiram o uso da visão de perto.

A esfera visual máxima alcançada pelas crianças participantes foi de $50 \mathrm{~cm}$. Essa informação é de extrema importância para os responsáveis e para os profissionais que acompanham as crianças, uma vez que todas as mensuraçôes e intervençôes devem ser realizadas dentro da esfera visual das mesmas. Entretanto, é importante ressaltar que o nível de interesse da criança pelo objeto pode apresentar interferência direta na sua resposta; dessa forma, pequenos objetos com apelo emocional podem causar respostas em uma esfera visual maior do que os objetos menos importantes que possuem o mesmo contraste e a mesma cor (HYVARINEN, 1995). Isso se justifica pelo fato de que a insistência em se mostrar determinada imagem ao cérebro pode desencadear interesse em vê-la novamente e favorecer o desenvolvimento de habilidades atencionais (DUTTON, 2002; LIM et. Al, 2005).

$\mathrm{O}$ adequado posicionamento das crianças foi fundamental para o bom desempenho na avaliação, tendo sido necessário realizar tentativas em diversas posiçóes com as crianças 1, 2 e 4. De acordo com a literatura, a avaliação deve incluir observaçóes dos efeitos de diferentes posturas sobre as funções óculo-motoras, a coordenação olho-mão e o papel dos reflexos anormais no uso da visão (HYVARINEN, 1995), considerando que o padráo postural de crianças com múltipla deficiência pode levar o examinador a conclusóes equivocadas se não forem cuidados os aspectos de organização da postura para otimização das respostas visuais (HYVARINEN, s/d; BRUNO, 2005; MARQUES \& MENDES, 2014).

As crianças 2, 3 e 4 demonstraram intenção motora para alcançar os objetos apresentados durante a avaliação. Hyvarinen (1995) afirma que as posturas mais eficazes na facilitação da coordenação olho-mão, bem como na inibição de posturas inadequadas, devem ser registradas e comunicadas para todos os envolvidos no cuidado com a criança, para que todos saibam o que esperar quando mudarem a criança de posição. Ademais, a existência de atividades reflexas requer uso cuidadoso de posturas e jogos situacionais que facilitem a coordenação da visão e os movimentos manuais das crianças com múltipla deficiência. 
Cabe ressaltar, entretanto, que a obtenção e a interpretação das respostas visuais dadas pelas crianças com múltipla deficiência são, geralmente difíceis, especialmente no caso de crianças com quadros neurológicos associados (NOBRE et. al, 1998), já que as alteraçôes oculares e centrais se somam e se confundem (NAKANAMI \& VASCONCELOS, 2011).

O quadro abaixo apresenta os resultados da avaliação da visão funcional, por meio do teste Lea Gratings.

Quadro 3 - Avaliação da visão funcional (Teste Lea Gratings)

\begin{tabular}{|c|c|c|c|c|c|c|}
\hline $\begin{array}{l}\text { Ciclos por centímetro } \\
\text { Distância }\end{array}$ & 0.25 & 0.50 & 1.00 & 2.00 & 4.00 & 8.00 \\
\hline Criança 1 & - & $29 \mathrm{~cm}$ & - & - & - & - \\
\hline Criança 2 & - & $29 \mathrm{~cm}$ & - & - & - & - \\
\hline Criança 3 & - & - & - & - & $57 \mathrm{~cm}$ & - \\
\hline Criança 4 & $29 \mathrm{~cm}$ & - & - & - & - & - \\
\hline Criança 5 & - & - & $29 \mathrm{~cm}$ & - & - & - \\
\hline
\end{tabular}

Quanto aos resultados da avaliação da visão funcional por meio da aplicação do teste Lea Gratings, verificou-se que, em todos os casos, foram apresentados valores bem abaixo dos valores médios de acuidade visual de grades binocular, de acordo com a faixa etária das crianças (SALOMÃO \& VENTURA, 1995). Isso pode estar relacionado ao fato de que apenas três crianças apresentavam diagnóstico conclusivo e nenhuma das crianças realizava ou havia realizado estimulação visual, apesar de não ser possível afirmar que as intervençôes precoces e terapêuticas não abordavam esse tipo de estimulação. É importante ressaltar que nenhuma das crianças participantes encontrava-se em período de desenvolvimento crítico da visáo, sendo possível inferir que as funçóes visuais podem náo ter sido adequadamente desenvolvidas.

\section{Informações/Orientações sobre a condição visual dos participantes}

Os responsáveis das crianças 2 e 3 declararam o recebimento de informaçôes/ orientações sobre a condiçâo visual das mesmas por médicos oftalmologistas; os responsáveis das crianças 3 e 4 declararam ter recebido informaçóes/ orientaçóes de outros profissionais da saúde. Os demais responsáveis referiram não ter recebido orientaçôes/ informaçôes acerca da condição visual das crianças.

Verificou-se que somente um professor acusou recebimento de informaçóes/ orientaçôes acerca da condição visual da criança 2, pelo professor do AEE. 


\section{Suporte Informacional}

Para apresentar os resultados da avaliação das crianças participantes para os responsáveis e os professores, foi elaborado um instrumento denominado suporte informacional. O suporte informacional visou se constituir como um instrumento de fácil visualização e compreensão pelos responsáveis e professores envolvidos com o processo de escolarização das crianças, porém, não foi objetivo do estudo a retroalimentação dos participantes acerca do instrumento.

Foi planejado para apresentar as informaçóes coletadas na avaliação da visão funcional, bem como para informar familiares, profissionais e comunidades sobre a importância da prevenção e detecção de alteraçôes visuais, em crianças com ou sem deficiência, e especificamente, nas crianças com múltipla deficiência, enfatizando a avaliação da visão funcional como instrumento adequado para o público-alvo dessa pesquisa. Dessa forma, pode ser considerado relevante para alertar e orientar profissionais, familiares e comunidade acerca da deficiência visual associada à múltipla deficiência, podendo ser considerado, até mesmo, um objeto de intervenção.

\section{Conclusão}

O presente estudo reafirmou a importância da avaliação da visão funcional para subsidiar a proposição de orientaçóes relevantes para o planejamento e realização de açôes e intervençóes dos responsáveis e dos professores com as crianças que compuseram a amostra.

A avaliação da visão funcional deveria complementar os resultados clínicos do exame oftalmológico, entretanto, os responsáveis possuíam pouca ou nenhuma informação sobre a condição visual das crianças. Dessa forma, no presente estudo, a avaliação da visão funcional ofereceu informaçóes importantes para levantar hipóteses sobre os tipos de alteraçóes visuais das crianças, bem como para subsidiar orientaçôes relacionadas ao desempenho das atividades cotidianas e pedagógicas para os responsáveis e para os professores.

Durante o desenvolvimento do trabalho, percebeu-se a importância de registrar os resultados da avaliação das crianças em um documento de fácil visualização e compreensão pelos responsáveis e professores envolvidos com o processo de escolarização das crianças.

Reforçou-se o direito e a importância da disponibilização do AEE para todos os níveis de ensino escolar.

Ficou evidenciado que, independentemente das limitaçóes e necessidades das crianças participantes no estudo, foram possíveis a realização de açôes que conduzem à ampliação das possibilidades de desenvolvimento das habilidades funcionais das mesmas.

\section{Referências}

ARAÓZ, S. M. M. Inclusáo de alunos com deficiência múltipla: análise de um programa de apoio. Tese de doutorado. Universidade Federal de São Carlos: UFSCar, 2010. 185 p. 
BRASIL. Lei no 9.394, de 20 de dezembro de 1996. Estabelece as diretrizes e bases da educaçáo nacional. Disponível em: <http://www.planalto.gov.br/CCIVIL_03/leis/L9394.htm>. [Acesso em 05 de julho de 2015].

BRUNO, M. M. G. Avaliaçáo educacional de alunos com baixa visão na Educaçáo Infantil: uma proposta de adaptação e elaboração de instrumentos. 2 vol. Tese (Doutorado em Educação). Marília: Faculdade de Filosofia e Ciências, UNESPP, 2005.

BRUNO, M. G. M. Educação Infantil: Saberes e Práticas da Inclusão: Dificuldades acentuadas de aprendizagem: Deficiência Múltipla. Brasília: MEC, Secretaria de Educação Especial, 2006. 58 p.: il.

BRUNO, M. M. G. Avaliaçáo educacional para alunos com baixa visão e múltipla deficiência na educaçáo infantil: uma proposta de adaptação e elaboração de instrumentos. GT: Educação Especial/ n. 15, Anped Nacional, Caxambu, 2008.

BRUNO, M. M. G. Avaliação funcional da visão e do desenvolvimento. In: SIAULYS, M. O. C.; ORMELEZI, E.; BRIANT, M. E. Deficiência visual associada á deficiência múltipla: encarando desafios e construindo possibilidades. São Paulo: Laramara, 2011, 304 p.

CARVALHO, E. N. S. Programa de capacitaçáo de recursos humanos do Ensino fundamental: Deficiência múltipla- Volume 1. Brasília: Ministério da Educação (MEC), Secretaria de Educação Especial, 2000.

COLENBRANDER, A. Aspects of Vision Loss - Visual Functions and Functional Vision. Visual Impairment Research - 2003, Vol. 5 No. 3, pp. 115-136.

CÔRREA, B. A. S. Refração em crianças. In: SCHOR, P; URAS, R; VEITZMAN, S. Óptica, refração e visão subnormal. Cultura Médica: Guanabara Koogan. $2^{\circ}$ ed. Rio de Janeiro, 2011.

DUTTON, G. Visual problems in children with damage to the brain. s.l.: Visual Impairment Research, 2002. pp. 113-21. Vol. 4 (2).

FÁVERO, E. A. G.; PANTOLA, L. M. P.; MANTOAN, M. T. E. Atendimento Educacional Especial: aspectos legais. In: FÁVERO, E. A. G.; PANTOLA, L. M. P.; MANTOAN, M. T. E. Atendimento Educacional Especializado: aspectos legais e orientação pedagógica. SEESP/SEED/MEC, Brasília-DF, 2007.

FAVILLA, M. et. al. Avaliação da visão funcional de uma criança prematura com cegueira congênita. Cad. Ter. Ocup. UFSCar, São Carlos, v. 22, n. 2, p. 429-434, 2014.

FIGUEIRA, M. M. A. Assistência fisioterápica à criança portadora de cegueira congênita. Revista Benjamim Constant.Artigo 2: Tema Fisioterapia. Edição 17, dez/2000.

GATO, M. I. T. P. et. al. Atençấo à saúde ocular de crianças com alterações neurológicas: Relevância da avaliação oftalmológica. Temas sobre Desenvolvimento 2008: 16(93):100-3.

GIL, A. C. Como elaborar projetos de pesquisa. 5. ed. São Paulo: Atlas, 2010. 184p.

HADDAD, M. A. O.; SEI, M.SAMPAIO, M. W. , KARA-JOSÉ,N. Causes of visual impairment in children: a study of 3.210 cases. J Pediatr Ophthalmol Starbismus, 2007; 44:232-40.

HADDAD, M. A. O.; SAMPAIO, M. W. Aspectos globais da deficiência visual. In: SAMPAIO, M. W. et al. Baixa visão e cegueira: os caminhos para a reabilitação, a educaçáo e a inclusão. Cultura Médica Guanabara Koogan, 2010; 7-16.

HOUNSTOUN-PRIME, C.; NAKAI, S. Distinguishing Novelty and Familiarity Effects in Infant Preference Procedures Carmel Infant and Child Development. Infant Child Dev. 2004; 13: 341-48.

HYVARINEN, L. Considerations and evaluation and treatment of the child with low vision. The American Journal of Occupational Therapy. Vol. 49, n. 9, out 1995.

HYVARINEN, L. Different visual functions. Disponível em: <www.lea-test.fi>. [Acesso em 20 de maio de 2015].

ILLET, G. Functional vision assessment. Optician, 2010. [Acesso em 08 de março de 2014]. Disponível em [opticianonline.net].

INTERNATIONAL COUNCIL EDUCATIONAL VISUAL IMPAIRMENT. World Health Organization Management of low vision children. Banglol: ICEVI-WHO, p. 23-24, july 1992.

ISAAC, D. L. C. et al. Prognostic Factors in Open Globe Injuries. Ophtalmol. 2003; 217:431-5.

LIM, M. et. al. Development of visual acuity in children with cerebral visual impairment. s.l. : (Reprinted) Arch Ophthalmol, Sep 2005. Vol. 123. 
Isabela Maria Reis Barbosa - Heloísa Gagheggi Ravanini Gardon Gagliardo - Marilda Moraes Garcia Bruno - Maria Elisabete Rodrigues Freire Gasparetto

MARQUES, L. C.; MENDES, E. G. O aluno com deficiência visual cortical: teoria e prática. São Carlos: EDUFSCar, 2014, 153 p

MAYER, D. L. et. al. Monocular acuity norms for the Teller acuity cards between ages one month and four years. Invest Ophthalmol and Vis Sci. 1995; 36: 671-685.

NAKANAMI, C.; VASCONCELOS, G. Abordagem clínica da criança com baixa visão. In: SCHOR, P.; URAS, R.; VEITZMAN, S. Óptica, refraçáo e visão subnormal. Cultura Médica: Guanabara Koogan. $2^{\circ}$ ed. Rio de Janeiro, 2011.

NOBRE, M. I. R. S. et al. Múltipla Deficiência e Baixa Visão. Rev. Neurociências 6(3): 111-113, 1998.

NOBRE, M. I. R. S. et. al. Atendimento de terapia ocupacional em serviço de visão subnormal: caracterização dos usuários. Rev. Benjamin Constant. 2009; 43.

PIMENTA, G. M. Uso do teste Lea Gratings para avaliação da acuidade visual de resolução de grades em lactentes normais. Dissertação de mestrado. Campinas, SP [s.n.], 2012.

REMIGIO, M. C. et. al. Achados oftalmológicos em pacientes com múltiplas deficiências. Arq. Bras. Oftalmol. 2006, 69(6):929-32.

ROVEDA, P. A. Pedagogia do significado: contribuiçōes à intervenção precoce em bebês com deficiência visual. Diss. (Mestrado em Educação) - Fac. de Educaçáo, PUCRS. Porto Alegre, 2006. 159 f.

SALOMÃO, S. R. Desenvolvimento da acuidade visual de grades. Psicol USP. 2007; (18) 2: 63-81.

SALOMÃO, S. R; VENTURA, D. F. (1995). Large scale population age norms for visual acuities obtained with Vistech/Teller acuity cards. Investigative Ophthalmology and Visual Science, 36, 657-670.

SIAULYS, M. O. C; ORMELEZI, E; BRIANT, M. E. Deficiência visual associada á deficiência múltipla: encarando desafios e construindo possibilidades. São Paulo: Laramara, 2011, 304 p.

SKOCZENSKI, A. M.; NORCIA, A. M. (2002). Late maturation of visual hyperacuity. Psychological Science, 13, 537-541.

TABUSE, M. K. U.; CRONEMBERGER, M. F. Alteraçôes oftalmológicas em crianças com paralisia cerebral. In: SAMPAIO, M. W.; HADDAD, M. A. O.; HELDER, A. C. F. (Org.). Baixa visáo e cegueira: os caminhos para a reabilitaçáo, a educaçáo e a inclusáo. Cultura Médica Guanabara Koogan, 2010, v. Cap.8, p. 71-78.

TOPOR, I. Functional vision assessment. Arizona: University of Arizona, 2009.

VEITZMAN, S. Visão subnormal. s.l. : Rio de Janeiro: Cultura Médica. São Paulo: CBO: CIBA Vision, 2000. Vol. Manuais Básicos / CBO (17).

WORLD HEALTH ORGANIZATION - Programme for the Prevention of Blindness- Management of low vision in children - Report of a WHO Consultation. Bangkok: WHO/PBL/93.27; 1992.

YUDCOVITCH L, LINDEN ME, MAEDA J, SHORE N. An evaluation of infant visual acuity using Lea Grating Paddles e Teller Acuity Cards. J Optom Vis Dev. 2004; 35(3/4): 224-29.

ZIMMERMANN, A. Avaliação da visão funcional infantil em serviço oftalmológico universitário. Tese (Doutorado). Campinas: UNICAMP, 2013.

\section{Notas}

${ }^{1}$ A deficiência visual é caracterizada pela perda total ou parcial da capacidade visual, resultante de fatores ambientais e orgânicos, podendo ser congênita ou adquirida (WHO, 1992).

${ }^{2}$ De acordo com Pimenta (2012, p. 70), o teste de acuidade visual de grades Lea Gratings "utiliza-se de 3 raquetes para apresentar as grades. Em cada raquete de grade a frequência da grade impressa é dada por ciclos por centímetro (cpcm), ou seja, o número de ciclos por centímetro da superfície".

\section{Correspondência}

Isabela Maria Reis Barbosa - Universidade Estadual de Campinas. Cidade Universitária Zeferino Vaz - Barăo Geraldo. CEP: 13083-970. Campinas, São Paulo, Brasil.

E-mail: isabela_mrb@hotmail.com - heloisa@fcm.unicamp.br-marildabruno@ufgd.edu.br - gasparetto@fcm. unicamp.br 
Avaliação da visão funcional em crianças com deficiência visual e múltipla deficiência como estratégia de apoio para professores e responsáveis

Correspondência em 03 de janeiro de 2018

Final em 20 de março de 2018

Revista Educação Especial | v. 31 | n. 61 | p. 387-404| abr./jun. 2018

Santa Maria

Disponível em: <https://periodicos.ufsm.br/educacaoespecial> 
